\title{
The Important Clinical Aspects of Diabetes Mellitus*
}

\author{
Kinori KosAKA**, M D
}

Diabetes mellitus is one of the most important disorders affecting human beings for many reasons. With the social, cultural and economic prosperity of mankind, prevalence of diabetes mellitus is on its way of constant increase with marked aggravation of clinical features. Although the prognosis of the patients with this disorder has been considerably improved owing to the progress in medicine, grave limitation is still present concerning social activity and longevity of the patients on account of its relentless complications.

Among many problems on the clinical aspects of diabetes mellitus, the author has selected the following; i) present concept of the disease in the new light or "what is diabetes?", ii) pathogenesis, especially in association with HLA antigen, iii) diagnosis based on the recent advances in basic investigations, and iv) treatment, especially suppression of the progression of vascular complications through long term control.

\section{Concept of Diabetes Mellitus}

The concept of diabetes mellitus has been revised to a large extent recently ${ }^{1-3)}$. What has changed is not the disease itself, but the definition or concept of disease based on the progress of medicine.

I) Diabetes mellitus as defined by Aretaeus-The classical historical concept

The first accurate description of diabetes mellitus was made by Aretaeus in the second century. Diabetes mellitus was defined as “a peculiar lethal disease with specific signs and symptoms, with a chronic course, finally ending in acute exacerbation." Although the actual changes in concept which reflects the progress of diabetology cannot be presented here, this disease had been characterized as a consumptive hereditary disease with glycosuria for a long time.

\section{II) Abnormal GTT and diabetes}

When blood sugar determination became available in the twentieth century, it became clear that glycosuria is a consequence of hyperglycemia, and an increase in blood sugar is marked and prolonged after glucose load in diabetics. Since similar curves were noted in conditions other than diabetes, it has been an accepted procedure all over the world to make the diagnosis of diabetes after excluding other conditions. It is still an open question whether or not the diabetes, being thus defined, and meeting certain arbitrary criteria is in reality a clinical entity.

\section{III) Present day concept of diabetes}

By analyzing the factors which modify the GTT, and by compiling the data about diabetes, diabetes mellitus as a clinical entity may be defined not as a synonym to an abnormality in GTT, but as a far more complex disease, which was meticulously described originally by Aretaeus, and has been steadily clarified with the progress in medicine. Diabetes mellitus is at present characterized by the following clinical

* Presented at the 74th Annual Meeting of the Japanese Society of Internal Medicine, April, 8, 1977, in Tokyo.

** Professor of the Third Department of Internal Medicine, Faculty of Medicine, University of Tokyo, Hongo, Tokyo, Japan. 
features :

1) a genetically conditioned disorder especially concerning the onset of the disease,

2) a course precipitated or worsened by internal or external predisposing and/or aggravating factors with characteristic signs and symptoms, finally leading to death in coma in severe cases,

3) extensive and specific metabolic disturbance based on the deficiency of insulin action,

4) distinct and characteristic stages of prediabetes, chemical and overt diabetes,

5) specific microangiopathy, i.e. retinopathy, nephropathy and neuropathy.

6) improvement by diet restriction and insulin administration.

A hypothetical process governing all these 6 features seems to be most essentially related to the fundamental mechanism of diabetes, and this would consequently define this disease more precisely. According to our data ${ }^{4)}$, insulin response to glucose load seems to be decreased through all the phases of diabetes, although the details still remain to be accurately defined.

The insulin responses during $100 \mathrm{~g}$ GTT in definitely diabetic patients were invariably smaller than normal (Fig. 1). Insulin response remained subnormal even when glucose tolerance was improved to normal types after successful treatment of diabetes. As the insulin response of diabetes was more markedly depressed in its initial phase, the ratio of the increment of blood insulin (in $\mu \mathrm{U} / \mathrm{ml}$ ) to that of blood sugar (in $\mathrm{mg} / \mathrm{dl}$ ) 30 minutes after glucose load ( $\Delta \mathrm{IRI} / \Delta \mathrm{BS}$ ) was characteristically decreased in diabetes, providing a useful index for subsequent analysis. $\triangle \mathrm{IRI} / \triangle \mathrm{BS}$ was usually lower than 0.5 in diabetes, while it was higher than 0.5 in normal subjects.

In various pathological states such as gastrectomy, corticosteroid treatment, simple obesity, hyperthyroidism, acute hepatitis, liver cirrhosis, myocardial infarction, unlike true diabetes, insulin responses during GTT were progressively enhanced when glucose tolerance was impaired to borderline and diabetic types, and $\Delta \mathrm{IRI} / \Delta \mathrm{BS}$ usually ex- ceeded 0.5 irrespectively of glucose tolerance. These relationships are clearly visualized when the sums of six insulin values during GTT ( $\Sigma$ IRI) are plotted against the sums of blood sugar values ( $\Sigma$ BS) (Fig. 2). In hyperthyroidism and gastrectomy, $\Sigma$ IRI became greater when $\Sigma$ BS was increased (i.e. glucose tolerance was more impaired). Similar relationships were also observed in other pathological states. In contrast, $\Sigma$ IRI remained low throughout the wide range of $\Sigma$ BS in definitely diabetic patients.

What are the insulin responses of patients who had normal FBS and no retinopathy but later become definitely diabetic? In 20 cases, their FBS rose and eventually exceeded $140 \mathrm{mg} / \mathrm{dl}$ during follow up period (Table 1). It is noteworthy that the initial $\Delta \mathrm{IRI} / \Delta \mathrm{BS}$ ratios in these cases were all less than 0.5 regardless of their types of glucose tolerance, normal, borderline or diabetic. With only a few exceptions, $\Delta \mathrm{IRI} / \Delta \mathrm{BS}$ ratio remained low during follow-up period, regardless of types of glucose tolerance. The initial $\Delta \mathrm{IRI} / \Delta \mathrm{BS}$ ratios were also lower than 0.5 in 19 cases who were not definitely diabetic but later developed retinopathy. These results indicate that this characteristically low insulin response during GTT is not a consequence of moderate or severe derangement of carbohydrate metabolism as represented by elevated FBS or of diabetic microangiopathy, but rather a more specific feature of diabetes preceding the development of overt diabetes.

Subjects whose parents are diabetics have high chance of getting diabetes in the future. In 57 such cases, 23 had normal, 15 had borderline and 19 had diabetic type glucose tolerance. The number of cases with $\Delta \mathrm{IRI} /$ $\Delta \mathrm{BS}$ less than 0.5 were 9,10 and 17 cases in each group respectively. The overall prevalence of low insulin responders was about $60 \%$, and it was fairly constant in each age group. When these results are expressed by $\Sigma$ BS- $\Sigma$ IRI plot, cases with $\Delta \mathrm{IRI} / \Delta \mathrm{BS}$ below 0.5 were distributed in the zone of definite diabetes, and those with $\Delta \mathrm{IRI} / \Delta \mathrm{BS}$ above 0.5 fell in the zone of various diseases other than diabetes (Fig. 3). When com- 

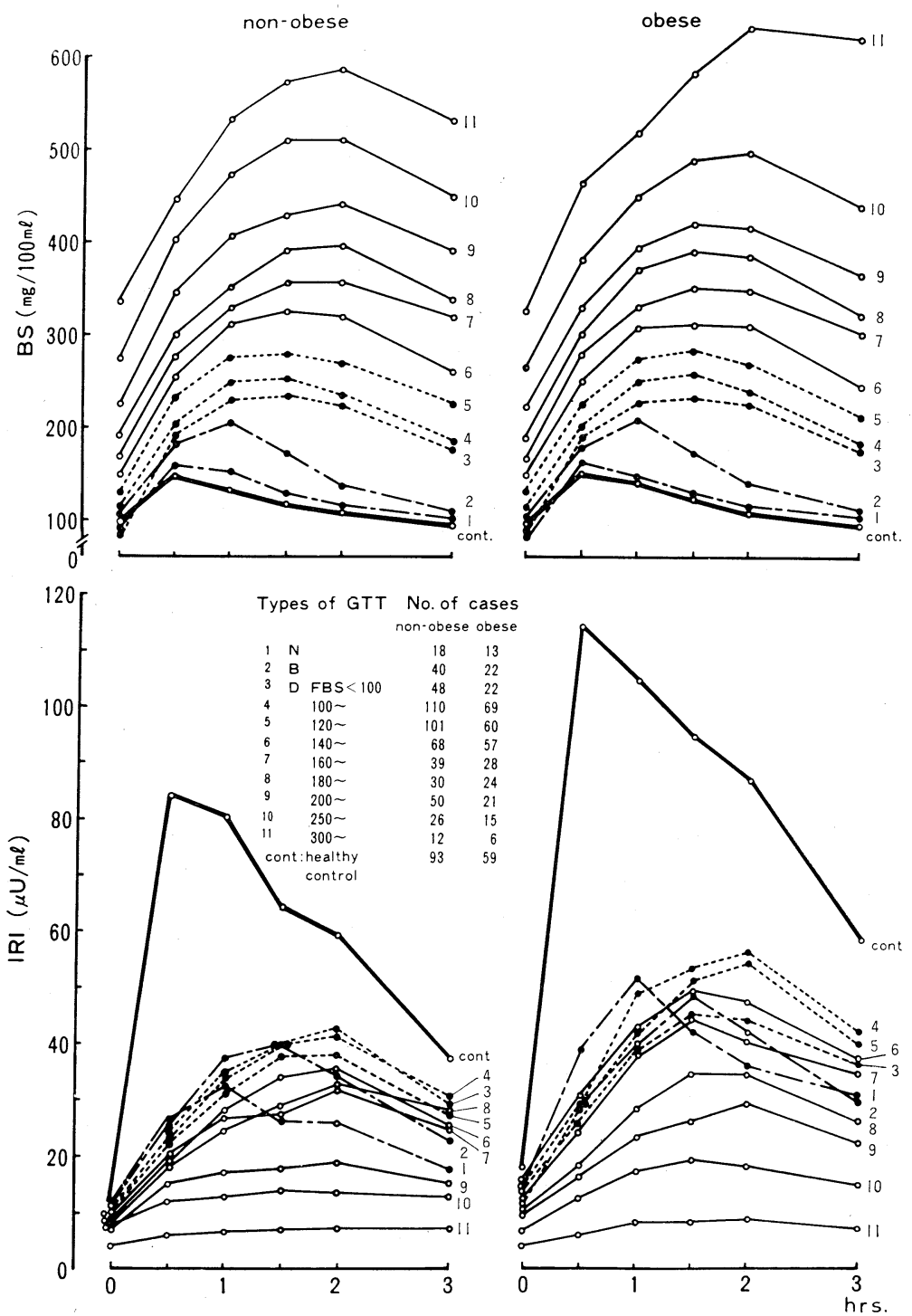

Fig. 1. Patterns of blood sugar and serum insulin concentrations (mean) during $100 \mathrm{~g}$ GTT in definite diabetics and healthy controls.

parisons were made between these subjects and definite diabetics with similar types of glucose tolerance, insulin response in two groups were similar.

These results strongly suggest that (1) the decrease in early insulin response during GTT is a specific feature of true diabetes, (2) this characteristically low insulin response exists throughout all phases of diabetes, from the prediabetic stage to complete remission of glucose tolerance in established diabetes. On the basis of such decrease in insulin response, most of the above physical features can be explained.

The important question still remaining in this respect is whether this decrease seen in most cases of "prediabetes" is in reality conditioned genetically. If this is the case, and the mechanism of onset of diabetes is explained on this decrease in insulin response, diagnostic definition and clinical concept of diabetes mellitus can be con- 


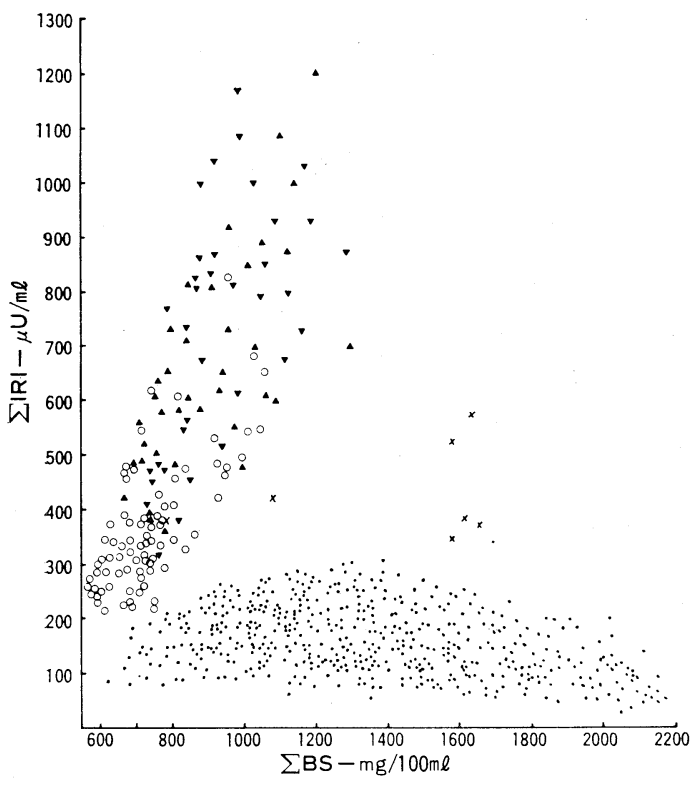

Fig. 2. Relationship between $\Sigma \mathrm{BS}(\mathrm{mg} / 100$ $\mathrm{ml})$ and $\Sigma$ IRI $(\mu \mathrm{U} / \mathrm{ml})$ during $100 \mathrm{~g}$ GTT in various pathological conditions (non-obese subjects).

A Hyperthyroidism (36), \ Gastrectomy (33), $\bigcirc$ Normal control (73), Diabetes mellitus (511), $\times$ Diabetes mellitus (7).

(numbers in parenthesis) sidered as one and the same.

\section{Histocompatibility Antigens in Diabetes}

As a novel immuno-gentic research field, HLA antigens have been studied in various disorders in relation to suspectibility ${ }^{5}$. We have performed HLA typing in 190 diabetics and 128 controls.

As shown in Table 2, among insulindependent diabetes (IDD) with onset less than 30 years of age, frequency of BW 54 and DYT were significantly higher. Both BW 54 and DYT are specific to Japanese and it is quite interesting that DYT is in linkage disequilibrium with BW 54. Also in IDD with onset 30 years and over, BW 54 was significantly more frequent $(\mathrm{P}<$ 0.003 , relative risk 5.1). Among insulinindependent diabetes, there was no significant difference in HLA frequency to controls irrespective of the age of onset.

These results suggest different genetic background and pathogenesis among groups of diabetcs classified based on insulin-dependency, giving a new clue for the elucidation of pathogenesis of diabetes.

Table 1. Types of $100 \mathrm{~g} \mathrm{GTT}$ and $\Delta \mathrm{IRI} / \Delta \mathrm{BS}$ in patients who had normal FBS and no retinopathy initially but later developed definite diabetes with FBS higher than $140 \mathrm{mg} / \mathrm{dl}$.

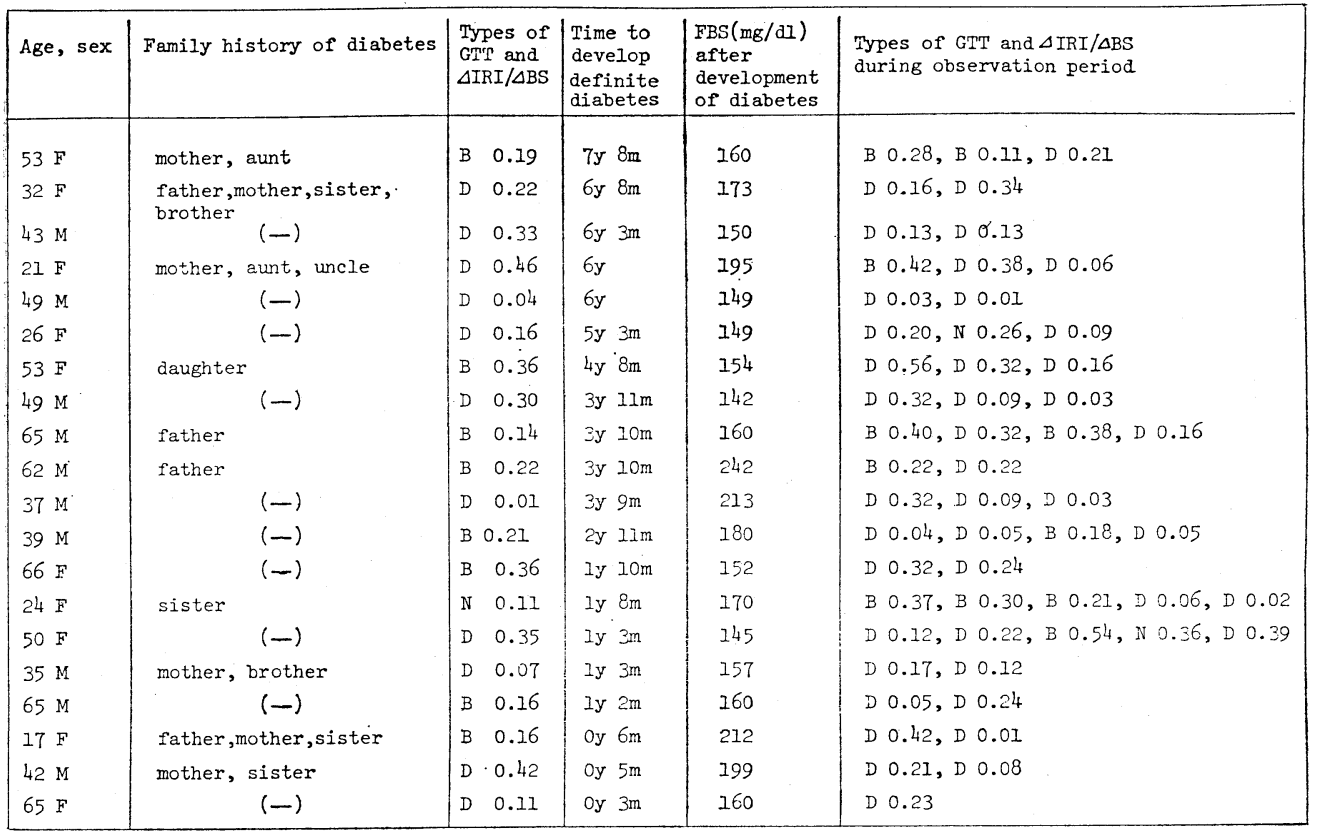



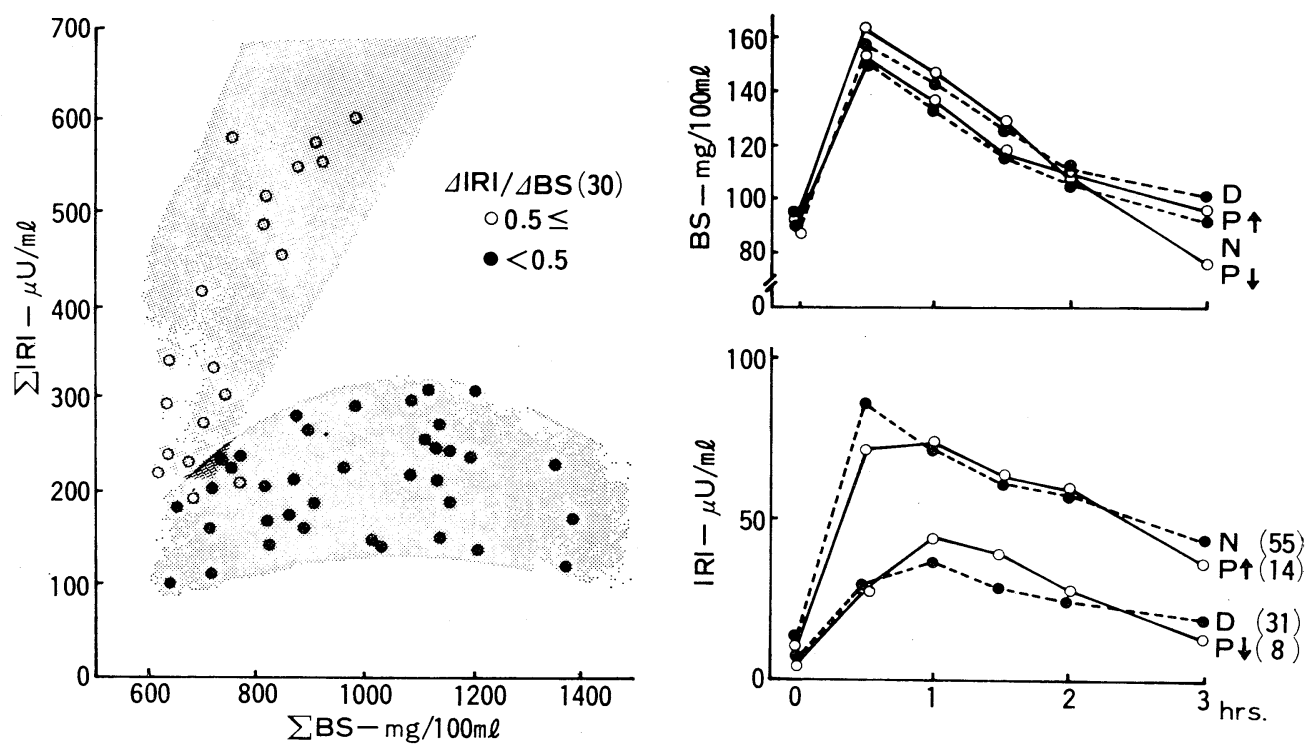

Fig. 3. Left: Relationship between $\Sigma$ BS $(\mathrm{mg} / 100 \mathrm{ml})$ and $\Sigma$ IRI $(\mu \mathrm{U} / \mathrm{ml})$ during $100 \mathrm{~g}$ GTT in subjects whose parents are both diabetic. Right: Patterns of blood sugar and serum insulin concentrations (mean) during $100 \mathrm{~g}$ GTT in subjects with normal glucose tolerance.

$\mathrm{D}$ : definite diabetes, $\mathrm{N}$ : healthy controls, $\mathrm{P} \uparrow:$ subjects whose parents are both diabetic and $\Delta \mathrm{IRI} / \Delta \mathrm{BS}$ are 0.5 or more, $\mathrm{P} \downarrow$ : subjects whose parents are both biabetic and $\Delta \mathrm{IRI} / \Delta \mathrm{BS}$ are less than 0.5 .

Table 2. HLA phenotype frequencies of Diabetes mellitus.

\begin{tabular}{|c|c|c|c|c|c|c|}
\hline \multirow[b]{2}{*}{ HLA } & \multirow{2}{*}{\multicolumn{2}{|c|}{$\begin{array}{l}\text { Control } \\
n=128\end{array}$}} & \multicolumn{4}{|c|}{ Insulin dependent } \\
\hline & & & $\begin{array}{c}<30 y r s . \\
n=77\end{array}$ & $x^{2}$ & $P$ & $\begin{array}{c}\text { Relative } \\
\text { Risk }\end{array}$ \\
\hline AI & ${ }_{3}^{\text {No. }}=$ & 2.3 & $\begin{array}{c}\text { No. } \\
1\end{array}$ & & & \\
\hline$A 2$ & $19 \quad 38$ & 38.3 & $39 \quad 50.6$ & 2.51 & & \\
\hline$A 9$ & 836 & 64.8 & $\begin{array}{ll}56 & 72.7\end{array}$ & 1.03 & & \\
\hline A 10 & $15 \quad !$ & 11.7 & $17 \quad 22.1$ & 3.16 & & \\
\hline$A \mid 1$ & $21 \quad 16$ & 16.4 & $\begin{array}{ll}6 & 7.8\end{array}$ & 2.41 & & \\
\hline$A W \mid 9$ & $25 \quad 15$ & 19.5 & $11 \quad 14.3$ & & & \\
\hline 85 & 4132 & 32.0 & $\begin{array}{ll}16 & 20.8\end{array}$ & 2.49 & & \\
\hline 87 & $21 \quad 16$ & 16.4 & 14.18 .2 & & & \\
\hline 88 & 0 & 0 & $2 \quad 2.6$ & & & \\
\hline$B 12$ & $16 \quad 12$ & 2.5 & $10 \quad 13.0$ & & & \\
\hline$B 15$ & $15 \quad 1$ & 1.7 & $8 \quad 10.4$ & 0.00 & & \\
\hline $8 w 22 \cdot 1$ & $5=$ & 3.9 & & & & \\
\hline BW35 & $24 \quad 18$ & 8.8 & $n=14.3$ & & & \\
\hline 840 & $46 \quad 35$ & 35.9 & $24 \quad 31.2$ & & & \\
\hline $8 W 54$ & $13 \quad 10$ & 10.2 & $35 \quad 45.5$ & 31.47 & 0.000 & 7.4 \\
\hline CWI & 443 & 34.4 & $5 / 2321.7$ & & & \\
\hline CW3 & $68 \quad 5$ & 53.1 & $15 / 2462.5$ & & & \\
\hline $\mathrm{CW}_{4}$ & 8 & 6.3 & $4 / 24 \quad 16.7$ & & & \\
\hline DYT & $11 / 651$ & 16.9 & $21 / 4645.7$ & 9.48 & 0.002 & 4.12 \\
\hline $\mathrm{DHO}$ & $11 / 691$ & 5.9 & $3 / 45 \quad 6.5$ & 1.49 & & \\
\hline
\end{tabular}

\section{Diagnosis of Diabetes Mellitus}

Recently, especially in Japan, attitude toward diagnosis of diabetes has been changing considerably ${ }^{6}$. The most important motive for this is the trend to rely on more specific and essential characteristics of diabetes rather than to depend on the glucose tolerance test which was hitherto presumed to be the best method of diagnosis of diaDetes. In this trend, it is hoped that the diseases which closely simulate diabetes can be differentiated from diabetes and the diagnosis can be make earlier. As stated above, including prediabetes, if insulin response to glucose load is decreased characteristically throughout all stages of diabetes, this decrease will serve as a sensitive and specific means for the differentiation between diabetes and similar situations.

Fig. 4 depicts plotting of $\Sigma$ BS- $\Sigma$ IRI relationship after $100 \mathrm{~g}$ GTT in non obese cases without overt diabetes. Cases with $\Delta \mathrm{IRI} / \Delta \mathrm{BS}(30 \mathrm{~min})$ less than 0.5 were distributed in diabetic area, while those with 0.5 and above were in non diabetic area 


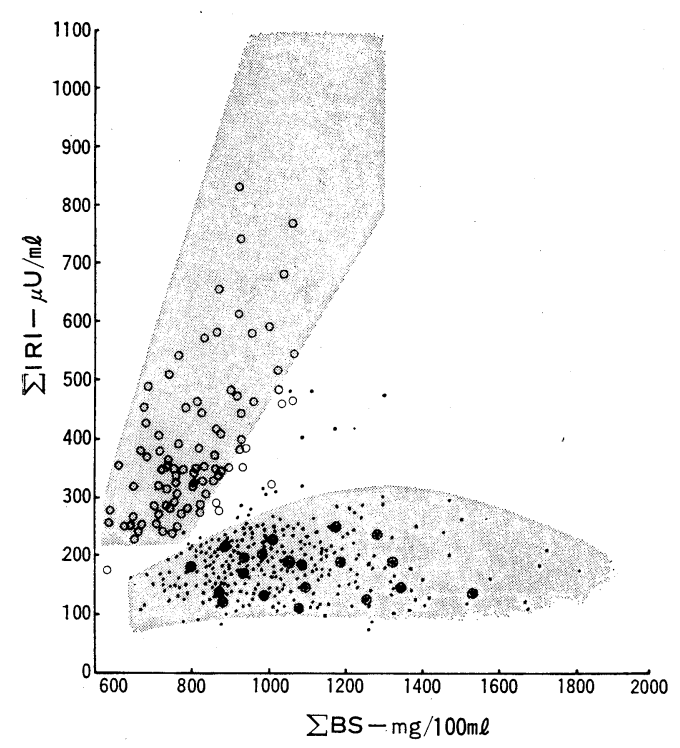

Fig. 4. Relationship between $\Sigma \mathrm{BS}(\mathrm{mg} / 100$ $\mathrm{ml})$ and $\Sigma$ IRI $(\mu \mathrm{U} / \mathrm{ml})$ during $100 \mathrm{~g}$ GTT in nonobese patients excluding definite diabetes.

$\Delta \mathrm{IRI} / \Delta \mathrm{BS}(30) \quad$ deviated from $\Sigma$ BS : $\Sigma$ IRI areas

- $<0.5320$ cases 18 cases $(5.8 \%$ )

○ $0.5 \leqq 89$ cases 9 cases $(10.1 \%)$

- developed overt diabetes during follow up period

fairly consistently with rather small number of exceptions. The results were similar in obese cases. When IRI response were grouped on the basis of GTT, cases with $\Delta \mathrm{IRI} / \triangle \mathrm{BS} 0.5$ and above were quite similar to normals and simple obesity, while cases less than 0.5 were quite similar to overt diabetics.

Among 1250 GTTs repeatedly performed in 446 definitely diabetic patients, only 15 tests $(1.2 \%)$ have $\Delta \mathrm{IRI} / \Delta \mathrm{BS}$ value higher than 0.5 in 13 patients (2.9\%). In patients other than those with definite diabetes, 934 GTTs were repeated in 330 cases, and their $\Delta \mathrm{IRI} / \Delta \mathrm{BS}$ were analyzed. $\Delta \mathrm{IRI} / \Delta \mathrm{BS}$ ratios were subdivided into two ranges; higher and lower than 0.5 . In 302 cases (91.6\%), $\Delta \mathrm{IRI} / \triangle \mathrm{BS}$ belonged to the same range on repeated tests. These results show that the reproducibility of $\Delta \mathrm{IRI} / \Delta \mathrm{BS}$ during GTT was high.

Also, when diabetics developed com- plicating disease which in itself augments insulin response despite decreased glucose tolerance, insulin response remained hyporesponsive.

From these results, when insulin response is decreased to the level of overt diabetes, the possibility of being a diabetic is to be considered seriously even if the glucose tolerance test itself gave a normal result. However, this does not mean that all the cases with insulin hyporesponsiveness to glucose should be diagnosed as diabetes. Also, it has not yet been made clear that an individual without insulin hyporesponsiveness can be free from diabetes throughout his life.

\section{Treatment of Diabetes and It's Problems}

The major aim of treatment of diabetes at present is the prevention of development of vascular complications closely related to this disease. Although it is generally considered that the incidence and progression of vascular complications in diabetic patients are prevented by the strict control of the diabetic state, the extent of possible achievement of the present treatment of diabetes was investigated in patients under regular follow-up for a long period, together with the problems inherent in the treatment.

\section{Vascular complications in diabetics under a long term follow-up}

This study conducted on 2025 patients who visited our diabetes clinic since its opening in 1957. Among them 627 and 318 patients have been regularly followed up for more than 5 and 10 years respectively. The fundamental principles of the treatment of diabetes mellitus in our clinic has been the total caloric restriction with well balanced diet, encouragement of daily physical exercise based on traditional methods and the use of therapeutic agents when indicated. The outline of the criteria of good control was to keep FBSs within normal range and to regulate the body weight to standard body weight or a little less than this. Maintenance of FBS within normal range usually keeps other parameters of 
specific diabetic metabolism parallelly within normal range. Regulation of body weight is important in order to decrease the degree of insulin deficiency substantially because obesity is one of the most important factors in precipitating and aggravating diabetes.

\section{(1) Diabetic Microangiopathy}

The frequency and the degree of diabetic retinopathy and proteinuria in patients who were not adequately treated at the time of the initial visit were higher in any age group when the FBS was higher and the duration was longer. This suggests that incidence and progression of microangiopathy is intimately related to metabolic disturbance of diabetes mellitus. The incidence and the progression of retinopathy and proteinuria after 5 and 10 years' followup was significantly decreased when time course of FBSs was better and body weight was well controlled (Fig. 5). There was no difference in the worsening of retinopathy

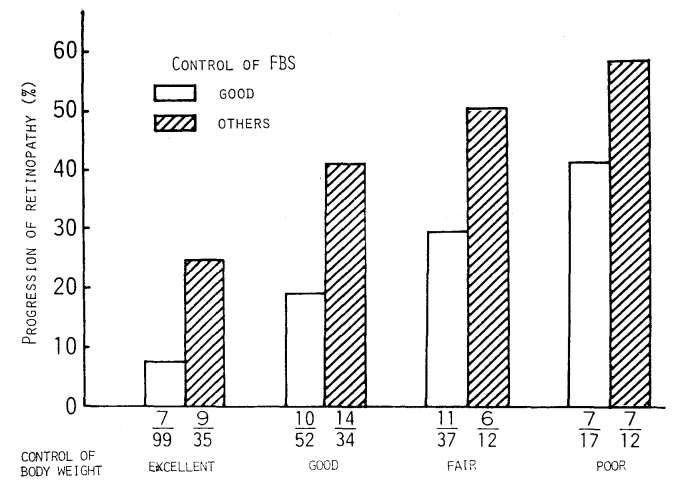

Fig. 5. Relationship of the progression of retinopathy to the controls of FBS and body weight (10 yrs follow up).

whatsoever among the different therapeutic agents employed when similar degree of control was obtained.

(2) Cardiovascular abnormality

In contrast to microangiopathy, the frequency of hypertension and the ECG abnormalities, mainly ST, $\mathrm{T}$ change, at the initial visit was not higher in patients with higher FBSs and longer duration. Hypertension was more frequent in higher age groups and, in the same age group, amongst the obese. Frequency of ECG abnormalities were higher in higher age groups and, in the same age group, in the hypertensive. Appearance of hypertension and progression of the EGG abnormalities after 5 and 10 years follow-up was not related to the time course of the FBS and the therapeutic agent (Fig. 6). The appearance of hypertension in the groups which failed to achieve

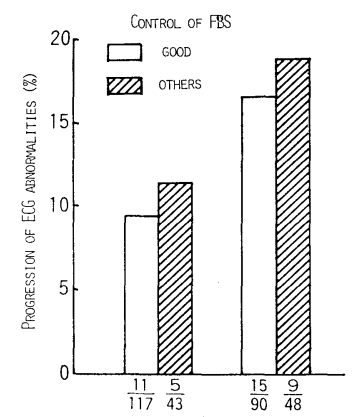

(-) (+)

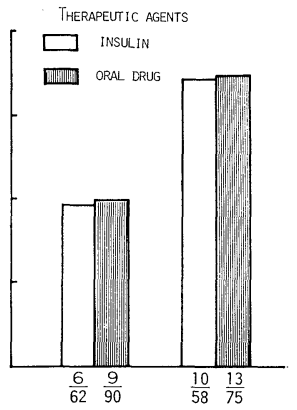

$(-)$

$(+)$
Fig. 6. Relationship of the progre ssion of ECG abnormalities to the control of FBS (left), and to therapeutic agents (right).

good weight control and the progression of EGG abnormalities in the hypertensive group were both significantly higher compared with the weight controlled and the normotensive patients respectively. However, there was no direct correlation between the progression of EGG abnormalities and weight control. These results do not necessarily mean that cardiovascular disease among diabetic patients in our country is not related to diabetic metabolism. However, this shows at least, that the cardiovascular disease is influenced much more by aging, obesity or hypertension than in case of microangiopathy. For the prevention of cardiovascular disease in diabetics, these results have to be taken seriously in to account.

\section{A few pathophysiological investigations}

Treatment of diabetes mellitus has been developed mainly based on the clinical experience of specialists in diabetes until now. The recent advance in the knowledge about diabetes and of the technology for biochemical research made a theoretical treatment of diabetes possible. From the stand point of alleviation of the deficiency of insulin 
action in all the cells, several pathophysiological investigations have been performed in diabetic patients followed up regularly for a long time.

(1) Insulin secretory capacity after long-term treatment

The remarkably decreased insulin response to glucose in untreated diabetics was definitely improved although within certain limits along with the improvement in glucose tolerance. This improvement which was similar in degree among the therapeutic agents employed can be maintained for a long period. Endogenous insulin response can not be demonstrated in brittle type diabetics on insulin treatment. The control of the body weight seems very effective in the alleviation of the deficiency of insulin action.

(2) Character analysis of insulin receptor of the peripheral monocytes

Although in patients with remarkable insulin resistance, e.g., extremely obese patients, decrease in the number of insulin receptor was demonstrated, the insulin receptor in diabetics in good control was not demonstrated to be different from normal control.

\section{(3) Lipids and lipoproteins in blood}

The blood levels of the triglyceride, the total cholesterol, the pre- $\beta$-lipoprotein, and the $\beta$-lipoprotein were all higher and $\alpha$ lipoprotein was lower in the groups of diabetics with higher FBS and obesity. When FBS was lowered and the weight was controlled, lipid values normalized.

Although there was no difference in the degree of improvement between the groups treated with diet alone and oral agents, it is to be remarked that in the insulin group, triglycerids and $\alpha$-lipoprotein decreased sometimes to a subnormal level.

\section{Prognosis and causes nf death after long-term follow-up}

The final aim of the control of diabetes is to achieve the normal way of life and the longevity equal to normals. The survey of the mortality among the patients with estimated duration longer than 5 years showed that the distribution of the age at death in patients (group A) regularly followed was higher compared with the patients (group B) who failed to do so. Although the percentage of cardiovascular causes in total deaths was quite similar between groups regularly followed up and those who failed to make regular visits, group A showed less death from renal causes while infarction and cerebral vascular accident were more common (Table 3). Between the insulin and the oral group, although the

Table 3. Causes of death among diabetic patients.

\begin{tabular}{ccc}
\hline Group* & A & B \\
\hline No. of cases & 120 & 84 \\
\hline Coma & $3.5 \%$ & $6.1 \%$ \\
Vascular & 60.0 & 57.8 \\
cardiac & 24.3 & 16.8 \\
cerebral & 24.4 & 19.2 \\
renal & 11.3 & 21.8 \\
Infections & 7.2 & 7.2 \\
Neoplasms & 18.3 & 18.1 \\
Others & 12.0 & 10.8 \\
Unknown & $(5)$ & $(1)$ \\
\hline
\end{tabular}

* See the text

numbers in parenthesis are cases whose causes of death are unknown, and they are excluded from calculation for per cent.

percentage of cardiovascular causes in total deaths was equal, death from renal causes was more common in the insulin group and cerebral vascular accident was more common in oral group. Although in patients with initial FBS less than $160 \mathrm{mg} / \mathrm{dl}$, no difference in mortality rate was noted between oral and insulin groups, when FBS exceeded $160 \mathrm{mg} / \mathrm{dl}$, insulin group had a higher death rate in both 5 to 10 and 10 to 18 years'. These results suggest that, on one hand, oral treatment does not give unfavourable influence on the mortality of the patients, and on the other hand, it seems to be very difficult to keep good control with insulin injection when indicated.

\section{CONCLUSIONS}

The clinical aspects of diabetes mellitus that are described here are based mainly 
on our recent results. The question of "what is diabetes?" has to be steadily pursued by deepening clinical and basic research as to the fundamental problem of diabetes mellitus. For this, the analysis of the genetic background of human diabetes and of the process by which the deficiency of insulin action is produced seems indispensable. The early and exact diagnosis of this disease will be established during this analysis.

The final aim of the treatment of diabetes is to eliminate the deficiency of insulin action at the cellular level. Whether this aim can be achieved by conventional treatment has to be investigated throughly in relation to the establishment of the criteria for proper control. After these criteria are established, the characteristics of the prognosis and the causes of death of diabetics will be equal to that of non-diabetics.

Since diabetes mellitus is quite variable in its age of onset and pathophysiology, the practice of the treatment has to be individually adjusted. Since the treatment has to be continued throughout the patients life, a major part of the treatment has to be carried out by the patients themselves. The importance of patient education de- pends on this fact. Furthermore, regular clinic visits including necessary examinations and observation of the appropriate directions requires not a little self restraint. The most indispensable requirement for success, therefore, is the mutual relationship and co-operation between the physician and his patients.

\section{REFERENCES}

1) Fajans SS: What is diabetes? Definition, diagnosis and course. Med Glin North Am 55 : 793, 1971.

2) Cerasi E, Luft R: Pathothysiology of diabetes. Diabetes Mellitus: Diagnosis and Treatment III, 1, 1971.

3) Kosaka K: What is diabetes? Progress and current state of concept of the disease. Tokyo J Med Sci $83: 1,1975$ (in Japanese).

4) Kosaka K, et al: Assay of blood insulin during $100 \mathrm{~g}$ GTT as a means for the diagnosis of diabetes mellitus. Jap J Med 16: 49, 1977.

5) Ritzmann SE: HLA antigens and disease associations. JAMA 236: 2305, 1976.

6) Kuzuya N, et al: Report of the Committee of the Diagnostic Criteria of the Oral Glucose Tolerance Test for Diabetes Mellitus-Recommendations on the evaluation of the oral glucose tolerance test for the diagnosis of diabetes mellitus. J Jap Diab Soc 13: 1, 1970 (in Japanese). 